\title{
A FAST AND RELIABLE MOTION HUMAN DETECTION AND TRACKING BASED ON BACKGROUND SUBTRACTION
}

\author{
B.S.M.MADHAVI ${ }^{1}$, M.V.GANESWARA RAO ${ }^{2}$ \\ ${ }^{I}$ PROJECT ASSOCIATE, DEPT OF ELECTRONICS AND COMMUNICATIONS, \\ ${ }^{2}$ ASSOC. PROFESSOR, DEPT OF ELECTRONICS AND COMMUNICATIONS, \\ SHRI VISHNU ENGINEERING COLLEGE FOR WOMEN, BHIMAVARAM \\ W.G.DT , ANDHRAPRADESH
}

\begin{abstract}
According to the result of moving object detection research on video sequences, this paper proposes a new method to detect moving object based on background subtraction. First of all, we establish a reliable background updating model based on statistical and use a dynamic optimization threshold method to obtain a more complete moving object. And then,morphological filtering is introduced to eliminate the noise and solve the background disturbance problem. At last, contour projection analysis is combined with the shape analysis to remove the effect of shadow, the moving human body are accurately and reliably detected. The experiment results show that the proposed method runs quickly, accurately and fits for the real-time detection. The occlusion is one of the most common events in object tracking and object centroid of each object is used for detecting the occlusion and identifying each object separately. Video sequences have been captured in the laboratory and tested with the proposed algorithm. The algorithm works efficiently in the event of occlusion in the video sequences.
\end{abstract}

Keywords-background subtraction; background model; moving object detection, shadow removal and occlusion detection.

\section{INTRODUCTION}

Human body motion analysis is an important technology which modem bio-mechanics combines with computer vision and has been widely used in intelligent control, human-computer interaction, motion analysis and virtual reality and other fields[ 1]. In which the moving human body detection is the most important part of the human body motion analysis, the purpose is to detect the moving human body from the background image in video sequences, and for the follow-up treatment such as the target classification, the human body tracking and behavior understanding, its effective detection plays a very important role. Currently, methods used in moving object detection are mainly the frame subtraction method, the background subtraction method and the optical flow method[2]. Frame subtraction method[3,4] is through the difference between two consecutive images to determine the presence of moving objects. Its calculation is simple and easy to implement. For a variety of dynamic environments, it has a strong adaptability,but it is generally difficult to obtain complete outline of moving object, liable to appear the empty phenomenon, as a result the detection of moving object is not accurate. Optical flow method[5,6] is to calculate the image optical flow field, and do clustering processing according to the optical flow distribution characteristics of image. This method can get the complete movement information and detect the moving object from the background better, however, a large quantity of calculation, sensitivity to noise, poor anti-noise performance,make it not suitable for real-time demanding occasions. The background subtraction method[7] is to use the difference method of the current image and background image to detect moving objects, with simple algorithm, but very sensitive to the changes in the external environment and has poor anti- interference ability. However,it can provide the most complete object information in the case of the background is known[8]. In this paper, in a single static camera condition, we combine dynamic background modeling with dynamic threshold selection method based on the background subtraction, and update background on the basis of accurate detection of object, this method is effective to enhance the effect of moving object detection.

In Computer Vision, object tracking is considered as one of the most important task. Various methods have been proposed and reported both in academia and industry for large number of real-time applications. The object tracking methods may broadly be categorized as template-based, probabilistic and pixel-wise. While the template-based method represents the object in a suitable way for tracking, the probabilistic method uses intelligent searching strategy for tracking the target object. Similarly, the similarity matching techniques are used for tracking the target object in pixel-based methods. However, among all the above said approaches, the template-based approach is found to be suitable for many real-time applications [1,2]. In this category of 
tracking methods, similarity of the predefined target is being calculated with the object translation. However, for object transformations such as translation, rotation and scaling this method often fails. This is due to the fact that the procedures of selection of target object as constant size templates. For handling this difficult issue, varying templates are used. The inclusion of background pixels into the template introduces the problem of positioning error and the positioning error continuously getting added while updating the template. In template based approach category, mean-shift method 3] and Kernel-based tracking method [4] have been proposed,where the color histograms of the target object is constructed Using a Kernel density estimation function. Since, the color Histogram is invariant feature for rotation, scaling and Translation, it is considered as one of the suitable feature for handling the problem of change in the scale, rotation and translation of target object. The object tracking is carried out by comparing the color histogram of the template and the target object. However, mean-shift method is not suitable for -D target object and monochromatic object. In case of monochromatic target object, even small variation in illumination, produces narrow histogram pattern and tracking often fails.

A very fundamental and critical task in computer vision is the detection and tracking of moving objects in video sequences. Possible applications are as follows (i) Visual surveillance: A human action recognition system process image sequences captured by video cameras monitoring sensitive areas such as bank, departmental stores, parking lots and country border to determine whether one or more humans engaged are suspicious or under criminal activity. (ii) Content based video retrieval: A human behavior understanding system scan an input video, and an action or event specified in high-level language as output. This application will be very much useful for sportscasters to retrieve quickly important events in particular games. (iii) Precise analysis of athletic performance: Video analysis of athlete action is becoming an important tool for sports ts training, since it has no intervention to the athletic. The main objective of this paper is to develop an algorithm that can detect human motion at certain distance for object tracking applications. We carry out various tasks such as motion detection, background modeling and subtraction, foreground detection, shadow detection and removal, morphological operations and identifying occlusion.

\section{BACKGROUND SUBTRACTION METHOD}

The background subtraction method is the common method of motion detection. It is a technology that uses the difference of the current image and the background image to detect the motion region[9], and it is generally able to provide data included object information. The key of this method lies in the initialization and update of the background image. The effectiveness of both will affect the accuracy of test results. Therefore, this paper uses an effective method to initialize the background, and update the background in real time.

\section{A. Background image initialization}

There are many ways to obtain the initial background image. For example, with the first frame as the background directly, or the average pixel brightness of the first few frames as the background or using a background image sequences without the prospect of moving objects to estimate the background model parameters and so on. Among these methods, the time average method is the most commonly used method of the establishment of an initial background[1O].

However,this method can not deal with the background image (especially the region of frequent movement) which has the shadow problems. While the method of taking the median from continuous multiframe can resolve this problem simply and effectively. So the median method is selected in this paper to initialize the background. Expression is as follows:

$\mathrm{B}_{\text {init }}(\mathrm{x}, \mathrm{y})=$ median $\mathrm{f}_{\mathrm{k}}(\mathrm{x}, \mathrm{y})$ where $\mathrm{k}=1,2,3, \ldots . \mathrm{n}(1)$

where $\mathrm{B}_{\text {init }}$ is the initial background $\mathbf{n}$ is the total number of frames selected.

\section{B. Background Update}

For the background model can better adapt to light changes, the background needs to be updated in real time, so as to accurately extract the moving object. In this paper, the update algorithm is as follows:

In detection of the moving object, the pixels judged as belonging to the moving object maintain the original background gray values, not be updated. For the pixels which are judged to be the background, we update the background model according to following rules: 
$\mathrm{B}_{\mathrm{k}+1}(\mathrm{x}, \mathrm{y})=$ beta $\mathrm{B}_{\mathrm{k}}(\mathrm{x}, \mathrm{y})+(1-$ beta $) \mathrm{F}_{\mathrm{k}}(\mathrm{x}, \mathrm{y})$

where beta belongs to $(0,1)$ is update coefficient, in this paper beta $=0.004, \mathrm{~F}_{\mathrm{k}}(\mathrm{x}, \mathrm{y})$ is the pixel grey value in the current frame. $\mathrm{B}_{\mathrm{k}}(\mathrm{x}, \mathrm{y})$ and $\mathrm{B}_{\mathrm{k}+1}(\mathrm{x}, \mathrm{y})$ are respectively the background value of the current frame and Next frame.As the camera is fixed, the background model can remain relatively stable in the long period of time. Using this method can effectively avoid the unexpected phenomenon of the background, such as the sudden appearance of something in the background which is not included in the original background. Moreover by the update of pixel gray value of the background, the impact brought by light, weather and other changes in the external environment can be effectively adapted.

\section{Occlusion Detection}

While two moving objects coming closer to each other, the background subtracted frame shows it as a single object. This situation is called as occlusion and will create problem while tracking two objects. In this approach, an algorithm is proposed for detecting the occlusion. This approach will inform the frame number where the occlusion has taken place. In the number of object in the frame is increased suddenly shows the entry of new objects into the frame or separation of occluded objects. Consequently if there is a sudden reduction of number of objects present in the frame indicates the process of occlusion of two or more objects or the exit of the objects from the frame to the outside area. This situation is experimented and is depicted in Fig. 1(a)-(d).

(a)
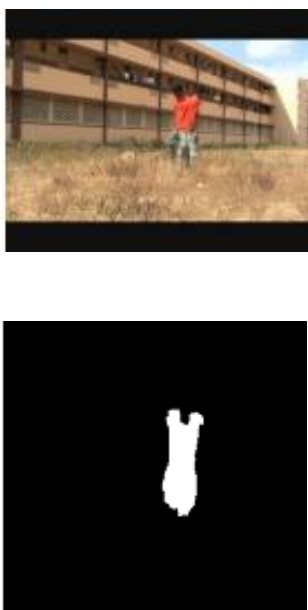

(b) (b)
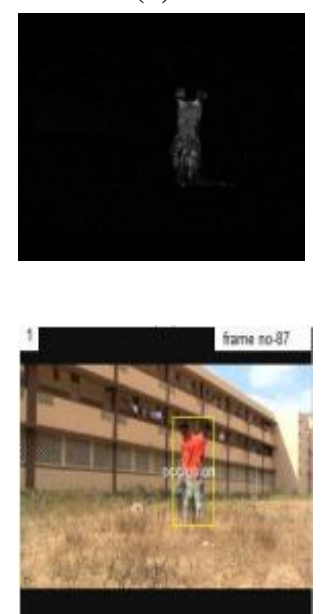

(d)

Fig1. Occlusion detection and background subtraction (a) Video Frame, (b) Background Subtraction, (c) BW Image Showing Objects and (d) Occlusion Detection

\section{A. Moving Object Extraction}

\section{MOVING OBJECT DETECTION}

After the background image $\mathrm{B}(\mathrm{x}, \mathrm{y})$ is obtained, subtract the background image $\mathrm{B}(\mathrm{x}, \mathrm{y})$ from the current frame $\mathrm{F}_{\mathrm{k}}(\mathrm{x}, \mathrm{y})$. If the pixel difference is greater than the set threshold $\mathrm{T}$, then determines that the pixels appear in the moving object, otherwise, as the background pixels. The moving object can be detected after threshold operation. Its expression is as follows:

$$
\begin{aligned}
D_{k}(x, y) & =\left\{1\left|F_{k}(x, y)-B_{k-1}(x, y)\right|>T ;\right. \\
& =0 \text { others }
\end{aligned}
$$

where $D_{k}(x, y)$ is the binary image of differential results. Delta $T$ is gray-scale threshold, its size determines the accuracy of object identification.

$$
\begin{aligned}
& \qquad T=\lambda \bullet \frac{1}{M \times N} \sum_{i=0}^{N-1} \sum_{j=0}^{M-1}|F(i, j)-B(i, j)| \\
& \text { Then } \\
& D_{k}(x, y)= \begin{cases}1 & \left|F_{k}(x, y)-B_{k-1}(x, y)\right|>T+\Delta T \\
0 & \text { others }\end{cases}
\end{aligned}
$$

As in the algorithm $\mathrm{T}$ is a fixed value, only for an ideal situation, is not suitable for complex

$$
\text { wWw.iosrjournals.org }
$$


environment with lighting changes. Therefore, this paper proposes the dynamic threshold method,we dynamically changes the threshold value according to the lighting changes of the two images obtained. On this basis, add a dynamic threshold delta $\mathrm{T}$ to the above algorithm. Its mathematical expression is as follows:

Where $\mathrm{A}$ is the inhibitory coefficient, set it to a value according to the requirements of practical applications, and the reference values is $2 . \mathrm{Mx} \mathrm{N}$ is the size of each image to deal with. $\mathrm{Mx} \mathrm{N}$ numerical results indicate the number of pixels in detection region. Delta $\mathrm{T}$ reflects the overall changes in the environment. If small changes in image illumination, dynamic threshold Delta $\mathrm{T}$ takes a very small value. Under the premise of enough pixels in the detection region, delta $\mathrm{T}$ will tend to $\mathrm{O}$. If the image illumination changes significantly, then the dynamic threshold !'1T will increase significantly. This method can effectively suppress the impact of light changes.

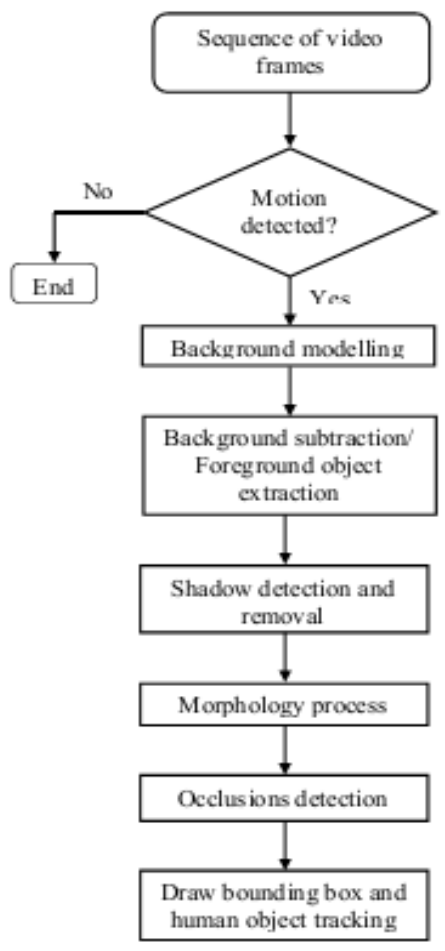

Fig1(a): Flow Chart for Human Object Tracking

Human motion analysis and detection are the foremost task in computer vision based problems. Human detection aims at segmenting regions corresponding to people from the entire image. It is a significant issue in human motion analysis system since the subsequent processes such as tracking and action recognition follows the motion detection. The motion detection and foreground object extraction algorithm consists of several sequential process es. The process algorithm is described in a flow chart and shown in Fig.1(a).

\section{B. Reprocessing}

As the complexity of the background, the difference image obtained contains the motion region, in addition, also a large number of noise. Therefore, noise needs to be removed. This paper adopts median filter with the 3 X3 window and filters out some noise. After the median filter, in addition the motion region, includes not only body parts, but also may include moving cars, flying birds, flowing clouds and swaying trees and other non-body parts. Morphological methods are used for further processing. Firstly, corrosion operation is taken to effectively filter out non-human activity areas. Secondly, using the expansion operation to filter out most of the non-body motion regions while preserving the shape of human motion without injury. After expansion and corrosion operations, some isolated spots of the image and some interference of small pieces are eliminated, and we get more accurate human motion region.

\section{Extraction of Moving Human Body}

After median filtering and morphological operations, some accurate edge regions will be got, but the region belongs to the moving human body could not be determined. Through observation,we can find out that when moving object appears, shadow will appear in some regions of the scene. The presence of shadow will affect the accurate extraction of the moving object. By analyzing the characteristics of motion detection,we 
combine the projection operator with the previous methods. Based on the results of the methods above, adopting the method of combining vertical with horizontal projection to detect the height of the motion region. This can eliminate the impact of the shadow to a certain degree. Then we analyze the vertical projection value and set the threshold value (determined by experience) to remove the pseudo-local maximum value and the pseudo-local minimum value of the vertical projection to determine the number and width of the body in the motion region, we will get the moving human body with precise edge. This article assumes that people in the scene are all in upright-walking state.

The flow chart of moving human body extraction is shown in Fig.2:

Human body detection is to identify the corresponding part of human from the moving region. But the extracted moving region may correspond to different moving objects, such as pedestrians, vehicles and other such birds, floating clouds, the, swaying tree and other moving objects. Hence we use the shape features of motion regions to further determine whether the moving object is a human being. Judging criteria are as follows:

(1) The object area is larger than the set threshold

(2) The aspect ratio of the object region should conform to the set ratio. If these two conditions are met, the moving object is the moving human body, or is not a human body

Figure2. The flow chart of moving human body extraction

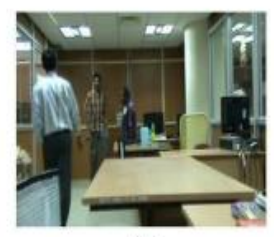

(a)

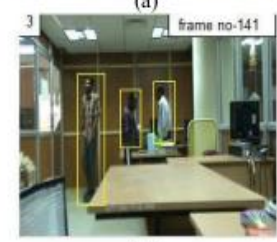

(d)

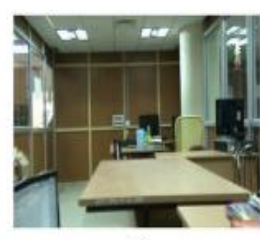

(b)

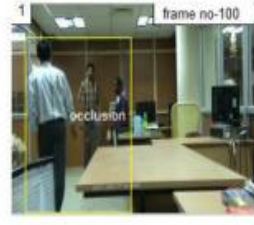

(e)

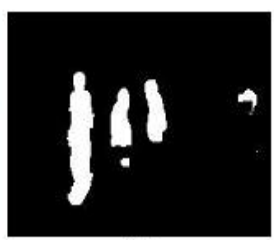

(c)

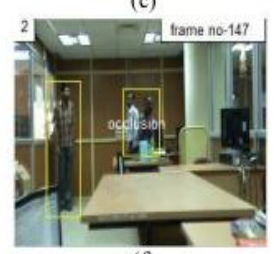

(f)

Figure2. Object tracking process (a) Video Sequences, (b) Background Model, (c) Background Subtraction, (d) Multiple Object Tracking with Bounding Box, (e) \& (f) Occlusion Detection

\section{EXPERIMENT}

The experimental results are presented to show that the proposed methods can achieve promising performance in background subtraction and foreground object extraction. This system detects and tracks the moving objects exactly. In this approach, the background scene is modeled using a set of background image frames, which basically consists of 5-30 consecutive frames. The object pixels are segmented out from its background followed by post- morphological process such as dilation and erosion to eliminate noisy pixels thus producing better results.

\section{A. Comparative results}

Kalman filter approach [26], algorithm is used for detecting and tracking the human objects in the video sequences. This algorithm is compared with the proposed approach for performance evaluation. Fig. 6(a) shows the tracking strategy of Kalman filter algorithm and (b) shows the tracking strategy of the proposed technique. While comparing the performance of Kalman filters and proposed approach, the following issues are observed.

(i) In most of the frames, the moving human objects are not tracked due to the static behavior of objects in the consecutive frames by Kalman filter algorithm. In the proposed approach, the background is frames. Thus, the background model helps the proposed approach to track the static human objects without failure.

(ii) The computation time for tracking the human objects in the video sequences using proposed approach is very low compared to Kalman filter algorithm. 
Fig.3. Performance comparison
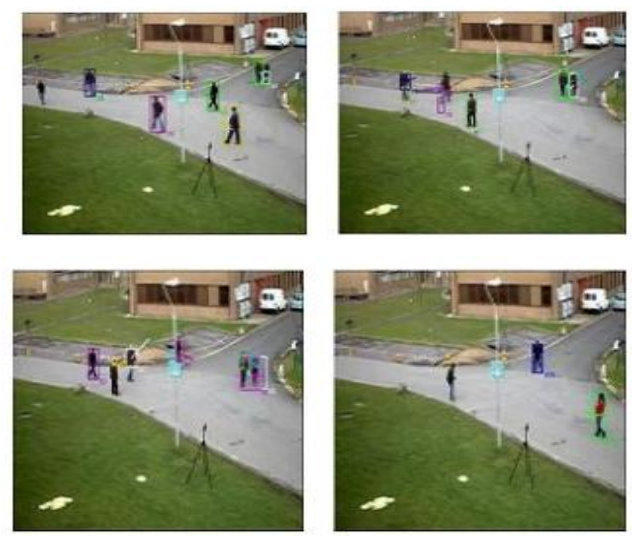

(a) kalman filter's results
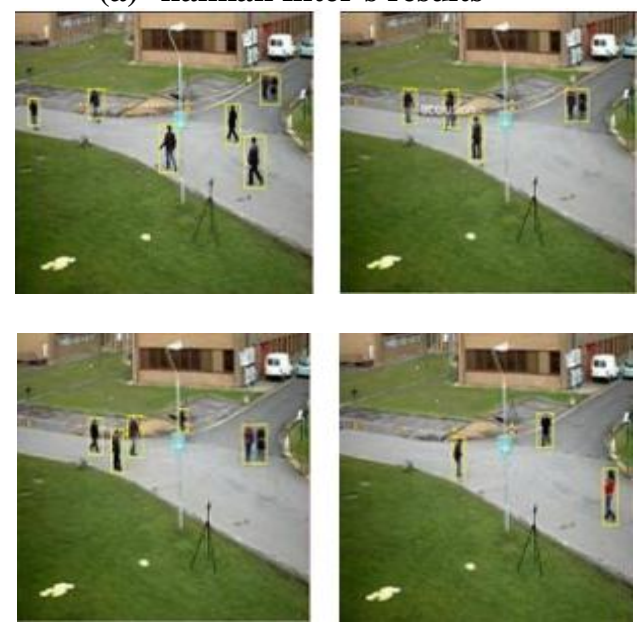

(b) proposed background subtraction \& shadow Removal

\section{CONCLUSION}

In this paper, an approach capable of detecting motion and extracting object information which involves human as object has been described. The algorithm involves modeling of the desired background as a reference model for later used in background subtraction to produce foreground pixels which is the deviation of the current frame from the reference frame. The deviation which represents the moving object within the analyzed frame is further processed to localized and extract the information. The occlusion has also been dealt effectively.

\section{REFERENCES}

[1] Crane H.D and Steele C.M.(1968) "Translation-Tolerant Mask Matching using Non coherent Reflective Optics Recognition Vol1.No2.,pp 129-136

[2] Grassl. C., Zinsser, T and Niemanr , H (2003) "Illumination Insensitive Template matching with Hyperplanes" , in proc . 25 $5^{\text {th }}$ pattern Recognition Symposium

[3] M.Dimitrijevic, "Human body pose detection using Bayesian spatial-temporal templates," 2007 International Conference on Intelligent and Advanced Systems, 2008, pp.764-9.

[4] Tao Jianguo, Yu Changhong, "Real-Time Detection and Tracking of Moving Object," Intelligent Information Technology Application,2008.UTA'08. Second International Symposium on Volume 2, 20-22 Dec.2008Page(s):860- 863

[5] Niu Lianqiang and Nan Jiang, "A moving objects detection algorithm based on improved background subtraction," Intelligent Systems Design and Applications, 2008. ISDA '08. Eighth International Conference onVolume3, 26-28 Nov. 2008Page(s ):604 607

[6] N.J.Bauerand P.N.Pathirana, "Object focused simultaneous estimation of optical flow and state dynamics," Intelligent Sensors, Sensor Networks and Information Processing, 2008. ISSNIP 2008. International Conference on15-18 Dec. 2008Page(s):61 - [71 Zhen Tang and Zhenjiang Miao, "Fast

[7] R.T. Collins, A. J. Lipton, H. Fujiyoshi, T. Kanade, Algorithms for Cooperative Multi sensor Surveillance, Proceeding of IEEE, Vol. 89. No.10, 2001

[8] I. Haritaoglu, D. Harwood, L.S. Davis, W4: Real-Time Surveillance of People and Their Activities, IEEE Transactions on Pattern Analysis and Machine Intelligence, Vol. 22, No.8, 2000. 
[9] A. Bobick and J. Davis, The Recognition of Human Movements Using Temporal Templates, IEEE Transactions on Pattern Analysis and Machine Intelligence, Vol. 23, No.3, March 2001.

[10] A. K. Jain, A. Ross, S. Prabhakar, An Introduction to Biometric Recognition, IEEE Transactions on Circuit and Sytems for Video Technology, Special Issue on Image- and Video-Based Biometrics, Vol. 14, No.1, pp. 4-20, January 2004.

[11] Ismail Haritaoglu, David Harwood, and Larry S. Davis, IW4: Who? When?Where? What? a Real-time System for Detecting and Tracking People," Proc.the third IEEE International Conference on Automatic Face and Gesture Recognition (Nara, Japan), IEEE Computer Society Press, Los Alamitos, Calif., 1998,pp.222-227.

[12] P.L.Rosin, Thresholding for Change Detection," Proceedings of International Conference on Computer Vision, 1998.

[13] N. Friedman, and S. Russell, Image Segmentation in Video Sequences: A Probabilistic Approach", Proceedings of the 13th Conference on Uncertainty inArticial intelligence, Morgan Kaufmann, 1997.

[14] C.R. Wren, A. Azarbayejani, T. Darrell, and A. Pentland, under: Real-time Tracking of the Human Body," IEEE Trans. on Pattern Analysis and Machine Intelligence, Vol.19, No.7, IEEE Computer Society Press, Los Alamitos, Calif., July 1997, pp.780-785.

[15] J. Ohya, et al., IVirtual Metamorphosis", IEEE Multimedia, April-June 1999.

[16] J. Davis, and A. Bobick, IThe representation and Recognition of Action using Temporal Templates", Proceedings of Conference on Computer Vision and Pattern Recognition, 1997.

\section{Authors:}

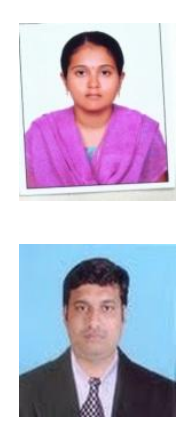

Ms.B.S.M.Madhavi pursuing M.Tech in VLSI Design Electronics and communication Engineering in Shri Vishnu Engineering College for Women from Jawaharlal Nehru TechnologicalUniversity,Kakinada.

Mr.M.V.GaneswaraRao pursuing Ph.d in VLSI Technology in Electronics and communication Engineering from Jawaharlal Nehru Technological university, Kakinada. He completed M.tech degree from Gudlavalleru Engineering college, Gudlavalleru and B.Tech in Electronics and communication Engineering for ASR Engineering college,TANUKU.Currently working as Assoc.Professor (sr) in shriVishnu Engineering college for women Bhimavaram (A.P). 